\title{
SNARE dynamics during melanosome maturation
}

\author{
Norihiko Ohbayashi ${ }^{* 1}$ and Mitsunori Fukuda ${ }^{\dagger 1}$
} *Department of Physiological Chemistry, Faculty of Medicine and Graduate School of Comprehensive Human Sciences, University of Tsukuba, 1-1-1 Tennodai, Tsukuba 305-8575, Japan

${ }^{\dagger}$ Laboratory of Membrane Trafficking Mechanisms, Department of Integrative Life Sciences, Graduate School of Life Sciences, Tohoku University, Aobayama, Aoba-ku, Sendai, Miyagi 980-8578, Japan

Running title: Role of SNAREs in melanosome maturation

${ }^{1}$ Address for correspondence: Norihiko Ohbayashi, Department of Physiological Chemistry, Faculty of Medicine and Graduate School of Comprehensive Human Sciences, University of Tsukuba, 1-1-1 Tennodai, Tsukuba 305-8575, Japan (e-mail: nohbayashi@md.tsukuba.ac.jp) or Mitsunori Fukuda, Laboratory of Membrane Trafficking Mechanisms, Department of Integrative Life Sciences, Graduate School of Life Sciences, Tohoku University, Aobayama, Aoba-ku, Sendai, Miyagi 980-8578, Japan (e-mail: nori@m.tohoku.ac.jp). 
Key words: BLOC, melanogenic enzymes, melanosome maturation, membrane traffic, Rab small GTPase, SNARE.

Abbreviations used: ANKR, ankyrin repeat; AP, adaptor protein; BLOC, biogenesis of lysosome-related organelles complex; GEF, guanine nucleotide exchange factor; HPS, Hermansky-Pudlak syndrome; KD, knockdown; LRO, lysosome-related organelle; SNAP, synaptosomal-associated protein; SNARE, soluble NSF ( $N$-ethylmaleimide-sensitive factor) attachment protein receptor; $t$-SNARE, target SNARE; Tyrp1, tyrosinase-related protein 1; VAMP, vesicle-associated membrane protein; Varp, VPS9-ankyrin-repeat protein; VID, VAMP7-interaction domain; VPS9, vacuolar sorting protein 9; $v$-SNARE, vesicle SNARE. 


\section{Abstract}

Historically, studies on the maturation and intracellular transport of melanosomes in melanocytes have greatly contributed to elucidating the general mechanisms of intracellular transport in many different types of mammalian cells. During melanosome maturation, melanosome cargoes including melanogenic enzymes (e.g., tyrosinase) are transported from endosomes to immature melanosomes by membrane trafficking, which must require a membrane fusion process likely regulated by SNAREs (soluble NSF ( $N$-ethylmaleimide-sensitive factor) attachment protein receptors). In this article, we review the literature concerning the expression and function of SNAREs (e.g., $v$-SNARE VAMP7 and $t$-SNAREs syntaxin-3/13 and SNAP-23) in melanocytes, especially in regard to the fusion process in which melanosome cargoes are finally delivered to immature melanosomes. We also describe the recent discovery of the SNARE recycling system on mature melanosomes in melanocytes. Such SNARE dynamics, especially the SNARE recycling system, on melanosomes will be useful in understanding as yet unidentified SNARE dynamics on other organelles. 


\section{Introduction}

The melanosomes in melanocytes are cell-type specific organelles in which melanin is synthesized and stored, and they are classified into four stages (I-IV) according to their morphological features and degree of melanin deposition. Melanosomes do not contain any pigment in the initial stage (I) but gradually darken as melanin is produced in the later stages (II-IV). The pigmentation process requires that three melanogenic enzymes, i.e., tyrosinase, Tyrp1 (tyrosinase-related protein 1), and Dct (dopachrome tautomerase; also called Tyrp2), be transported to the melanosomes. Tyrosinase and Tyrp1 that have been properly folded in an endoplasmic reticulum and the Golgi apparatus are secreted by the trans-Golgi network and transported to melanosomes via endosomes. In addition to these melanogenic enzymes, non-enzymatic melanosome proteins such as a copper ion transporter ATP7A and a chloride ion channel OCA2 are also necessary to be properly transported to melanosomes for melanin synthesis. Thus, the intracellular endosomal system plays a pivotal role in the transport of these melanosome cargoes, and abnormalities in the transport system cause certain hereditary diseases characterized by impaired pigmentation of hair, skin, and eyes (e.g., Hermansky-Pudlak syndrome [HPS]) [1]. HPS (HPS1-9) is an autosomal recessive disorder characterized by oculocutaneous albinism and bleeding tendency. Their pathological effects are attributable to impaired biogenesis of lysosome-related organelles (LROs) in multiple cell types such as melanosomes in melanocytes [2]. Griscelli syndrome and Chédiak-Higashi syndrome are also known as LRO disorders characterized by hypopigmentation. Melanosome transport along the actin 
cytoskeleton is impaired in melanocytes from Griscelli syndrome patients, whereas melanosome biogenesis is impaired in melanocytes from Chédiak-Higashi syndrome patients [3].

Recent advances in live-cell imaging techniques have made it possible to analyze the dynamics of intracellular molecules more precisely, and, among other things, they have revealed that melanosome cargoes, including Tyrp1, are transported to melanosomes via recycling endosomes, which are specialized endosomes for sorting and recycling internalized plasma membrane proteins and lipids in general (Figure 1). Since melanosomes are surrounded by a lipid bilayer, the same as other organelles, transport of melanosome cargoes to melanosomes must be tightly regulated by a group of molecules that control membrane (or vesicular) trafficking: Rab small GTPases, adaptor protein (AP) complexes, and SNAREs (oluble NSF ( $N$-ethylmaleimide-sensitive factor) attachment protein receptors). In this article, we briefly review the literature regarding the known melanosome cargo transport pathways and summarize current knowledge concerning the role of SNAREs in melanosome biogenesis, especially in regard to the fusion process between transport vesicles that contain melanosome cargoes and maturing (or immature) melanosomes.

\section{Transport pathways of melanosome cargoes to maturing melanosomes}

Two main pathways, both of which utilize the endosomal system, have been reported to be involved in the transport of melanosome cargoes: one is a biogenesis of lysosome-related organelles complex (BLOC)-1-dependent pathway and the other is a 
BLOC-1-independent/AP-3-dependent pathway (Figure 1); however, BLOC-1 is known to cooperate with AP-3 in the transport of certain cargoes $[4,5] . \quad$ In a steady state, BLOC-1 and AP-3 are localized at distinct microdomains of early endosomes and are thought to transport different melanosome cargoes [5]. A BLOC is a protein complex composed of several subunits, and three types of BLOCs have been reported. BLOC-1, -2 , and -3 are well conserved protein complexes in metazoans. BLOC-1 is composed of at least eight subunits, and mutations in one of several of its subunits have been reported in HPS patients and in HPS model mice [6]. Loss of function of one of the three BLOCs, BLOC-1, is known to cause the severest pigment dilution (or coat color dilution) phenotype. Moreover, based on the results of creating double recessive or triple recessive mutant mice for each type of BLOCs, BLOC-1 has been proposed to function most dominantly in the pigment dilution phenotype [6]. BLOC-1 regulates the transport of certain melanosome cargoes, including ATP7A and Tyrp1, and promotes cargo transport from recycling endosomes to melanosomes $[7,8]$. Thus, BLOC-1 is closely related to the dynamics of recycling endosomes, consistent with a report that BLOC-1 and the recycling endosomal regulator Rab11 are genetically correlated in Drosophila [9]. Interestingly, BLOC-1 is not significantly involved in the tyrosinase trafficking to melanosomes. Actually, a certain amount of tyrosinase, but not of ATP7A copper transporter, is transported to melanosomes in BLOC-1-deficient melanocytes, but tyrosinase is unable to function, because it needs copper ions as a cofactor to exert its function [7]. Since no known protein motif has been found in each subunit of BLOC-1, the precise molecular mechanism regulated by BLOC-1 is still 
poorly understood. In recent years Raposo's group has reported that BLOC-1 controls the formation of Tyrp1-containing tubules, namely, the formation of recycling endosomes from early endosomes, by coordinating microtubules and actin filaments in the vicinity of the early endosomes [10]. BLOC-2 has been shown to function downstream of BLOC-1 and to promote fusion between recycling endosomes that contain melanosome cargoes and maturing melanosomes [11].

APs are tetrameric protein complexes that mediate the sorting of selective cargoes into vesicles, and five APs have been reported. One of them, AP-3, plays a pivotal role in the endosome trafficking system. The AP-3-dependent pathway is mainly used for tyrosinase transport to melanosomes, whereas the BLOC-1-dependent pathway is used for transport of ATP7A and Tyrp1 as mentioned above. On the other hand, transport of OCA2 to melanosomes requires both pathways, although it is much less dependent on BLOC-2, suggesting that more complicated transport pathways exist for transport of melanosome cargoes. [7, 8, 11-14].

\section{SNAREs in melanocytes}

A membrane fusion process between recycling endosomes and melanosomes is necessary for melanosome cargoes on recycling endosomes to be finally transported to melanosomes, and SNAREs, general fusion machineries, are likely to be involved in the process. SNAREs are small proteins consisting of 100 to 300 amino acids that are found in all eukaryotes, and each SNARE contains one or two SNARE motifs consisting of about 60 amino acids. Each SNARE motif consists of a coiled-coil 
structure containing repeating sequences of seven amino acids and forms an $\alpha$-helical structure upon formation of a complex with cognate SNAREs. Approximately 40 SNAREs have been identified in mammals thus far, and each SNARE is localized at different organelles within cells. A "SNARE hypothesis", which states that cognate SNAREs associate and assemble together to promote fusion, has been proposed, and membrane fusion has been demonstrated to occur only when one $v$-SNARE (vesicle SNARE) protein is present on the vesicle and two or three $t$-SNARE (target SNARE) proteins are present on the target membrane [15]. The SNARE complex is a bundle of four $\alpha$-helices, and, in principle, one $\alpha$-helix is supplied by each SNARE, however, in the case of the SNAP (synaptosomal-associated protein)-25 family members (SNAP-23, $-25,-29$, and -47 ), two helices are supplied by one SNAP. Crystallographic analysis has revealed that the bundle of four helices of the SNARE motif consists of 16 layers (named layers -7 to +8 along the bundle), and almost all of the layers are composed of hydrophobic amino acids, but, exceptionally, only the central layer (layer 0) is composed of glutamine (Q) or arginine (R) (i.e., polar residues), which enables the formation of hydrogen bonds between them. These amino acids are well conserved, and SNARE proteins containing arginine are called R-SNAREs while those containing glutamine are called Q-SNAREs. Interestingly, R-SNAREs and Q-SNAREs function as $v$-SNAREs and $t$-SNAREs, respectively [16].

The presence of SNAREs in melanocytes was originally reported by Wade et al. and Scott et al. They showed that various SNAREs are expressed in B16 melanoma cells $[17,18]$. Although the function of most of the reported SNAREs 
remains to be determined, one of the $v$-SNAREs, VAMP7 (vesicle-associated membrane protein 7; also known as TI-VAMP) has been reported to be involved in melanosome maturation. VAMP7 was originally described as a $v$-SNARE protein expressed in late endosomes and lysosomes, and, in melanocytes, it is clearly localized on melanosomes $[19,20]$. Two $t$-SNAREs, syntaxin-3 and syntaxin-13, are localized on melanosomes and tubular recycling endosomes that contain Tyrp1, respectively, in melanocytes, [11, $21,22]$, and another $t$-SNARE, SNAP-23, has been shown to be present in the melanosome fraction [22]. Possible involvement of $t$-SNARE syntaxin-17 in the age-related vitiligo-like coat color dilution in Gray horses has also been reported, but it is still unclear whether syntaxin-17 directly regulates melanosome cargo trafficking [23].

Transfer of melanogenic enzymes to melanosomes - anterograde trafficking regulated by SNAREs -

As described above, melanosome cargoes are transported to melanosomes via tubular recycling endosomes. BLOC-1 regulates tubulation of recycling endosomes by coordinating microtubule-dependent transport and local actin filament formation, and the resulting tubular recycling endosomes deliver melanosome cargoes by fusing with melanosomes. Thus, BLOC-1 is primarily thought to be an essential protein complex for anterograde transport of melanosome cargoes to melanosomes. Although syntaxin-13 is known to regulate cargo protein recycling to the plasma membrane and homotypic endosomal fusion in non-melanocytic cells $[24,25]$, it has been shown to 
serve as a $t$-SNARE localized at tubular recycling endosomes in melanocytes. Since Tyrp1 was found not to be transported to melanosomes and instead to be mislocalized to syntaxin-13-positive endosomes in BLOC-1-deficient melanocytes, BLOC-1 and syntaxin-13 are likely to cooperatively regulate Tyrp1 transport [8, 19]. Consistent with this, pallidin (BLOC1S6), a subunit of BLOC-1, has been shown to physically interact with syntaxin-13 [26]. VAMP7 is localized at melanosomes, and transport of VAMP7 to melanosomes is BLOC-1-dependent [19]. Moreover, VAMP7-knockdown (KD) melanocytes showed hypopigmentation in addition to Tyrp1 transport defects [19, $20]$, and the presence of its cognate $t$-SNARE proteins is necessary for VAMP7 to carry out its function in melanocytes. Syntaxin-13 is a likely candidate for the cognate $t$-SNARE, because it is known to form a complex with VAMP7 [27]. Actually, both Tyrp1 and VAMP7 mislocalize to lysosomes and a hypopigmentation phenotype is observed in syntaxin-13-KD melanocytes, suggesting that syntaxin-13 is involved in anterograde transport of melanosome cargoes [21]. On the other hand, Chi et al. comprehensively searched for molecules that are expressed in melanosomes by performing a proteomic analysis, and they identified syntaxin-3, another $t$-SNARE, in the melanosome fraction [28]. Yatsu et al. have determined that syntaxin-3 is clearly localized at melanosomes in melanocytes, and they have shown that both Tyrp1 transport to melanosomes and pigmentation are impaired in syntaxin-3-deficient melanocytes [22]. Similar defects have also been observed in melanocytes deficient in SNAP-23, another $t$-SNARE that is also present in the melanosome fraction [22]. Furthermore, the existence of a complex consisting of VAMP7-syntaxin-3-SNAP-23 in 
melanocytes supports a model in which this SNARE complex promotes Tyrp1 transport to melanosomes [22]. At least two types of SNARE complexes, VAMP7-syntaxin-13-unknown-t-SNARE(s) and VAMP7-syntaxin-3-SNAP-23, are involved in the transport of melanosome cargoes to melanosomes. However, it is still impossible to clearly explain why these two distinct SNARE complexes simultaneously function to transport melanosome cargoes in melanocytes. There may be a complicated mechanism(s) by which VAMP7 changes its cognate $t$-SNAREs spatiotemporally for melanosome cargo transport.

Rab small GTPases are essential regulators of various membrane trafficking processes that are conserved in all eukaryotes. They function as molecular switches by cycling between GDP-bound inactive and GTP-bound active states and promote membrane trafficking through interaction with their effectors (i.e., active Rab-binding partners) [29]. Interestingly, several Rabs and their effectors strongly influence the above-mentioned melanosome cargo transport regulated by SNAREs. The generally recognized mild coat color dilution of chocolate mice has been shown to be caused by dysfunction of Rab38 [30]. Rab38 and its close homolog Rab32 have been shown to be localized at early endosomes and mature melanosomes in melanocytes, and the two Rabs redundantly regulate tyrosinase and Tyrp1 transport to melanosomes [31-33]. Several effector molecules for Rab32/Rab38 have been identified, and functions of one of them, Varp (VPS9-ankyrin-repeat protein) in melanocytes, have been well studied [34]. Varp contains an N-terminal VPS9 (vacuolar sorting protein 9) domain and C-terminal tandem ANKR (ankyrin repeat) domains (named ANKR1 and ANKR2). 
The Varp VPS9 domain functions as a GEF (guanine nucleotide exchange factor) for Rab21, but Rab21 activation by Varp is not essential for Tyrp1 transport in melanocytes [20]. By contrast, ANKR1 specifically binds to the GTP-bound active Rab32/Rab38, and the ANKR1-Rab32/Rab38 interaction is essential for Tyrp1 transport. Furthermore, ANKR2 has been reported to indirectly regulate Tyrp1 transport through quality control of Varp protein [35, 36]. The region (VID: VAMP7-interaction domain) between the two ANKRs is essential for binding to VAMP7 [37], and VID-deleted Varp is unable to promote Tyrp1 transport to melanosomes [20]. Moreover, Di Pietro's group has reported that myosin Vc and BLOC-2 are other Rab32/Rab38 effectors. Myosin Vc has been shown to localize at early endosomes in melanocytes, where activated Rab32/Rab38 are present, and the interaction between myosin Vc and Rab32/Rab38 has been proposed to regulate trafficking of Tyrp1 and VAMP7 to melanosomes [31]. As mentioned above, BLOC-2 has been shown to participate in targeting melanogenic enzymes from recycling endosomes to melanosomes [11]. The above reports taken together indicate that Rab32/Rab38 and their effectors contribute to anterograde transport of melanosome cargoes to melanosomes through physically and/or functionally interacting with VAMP7.

\section{Recovery of SNAREs from melanosomes - retrograde trafficking of SNAREs -}

After fusion, the SNAREs that have been transported to melanosomes need to be retrieved in order to prepare the next round of melanosome cargo transport. Although VAMP7 has been reported to be internalized from the plasma membrane in an ArfGAP 
Hrb-dependent manner and to be returned to late endosomes via early endosomes [38], the SNARE recovery mechanisms in intracellular organelles are poorly understood. Marks' group has recently demonstrated part of the SNARE recovery mechanism from melanosomes by using fine live-cell imaging [19]. In a steady state, they found that melanosome cargoes such as Tyrp1 and OCA2 continue to localize on the melanosome membrane, whereas VAMP7 localizes to tubular structures formed from melanosomes, where Tyrp1, OCA2, and syntaxin-13 are not present, implying that VAMP7 is transported from melanosomes via a syntaxin-13-independent pathway (Figure 1). Interestingly, both Rab38 and Varp are localized in this VAMP7-positive tubule, and the binding to VAMP7 and Rab38 determines the Varp localization on the tubule. Since Tyrp1 is not loaded onto the VAMP7-positive tubule, the tubule is assumed to be a structure that recovers VAMP7 from melanosomes and returns it to early endosomes. BLOC-3 is a heterodimeric complex consisting of HPS1 and HPS4 and has been shown to be a Rab32/Rab38-GEF [39]. The recruitment of Rab32/Rab38 effector Varp to melanosomes is substantially reduced in BLOC-3-deficient melanocytes, where Rab32/Rab38 are not activated, and formation of VAMP7-recycling tubules from melanosomes is also suppressed. Moreover, Owen's group has clearly demonstrated that the interaction between VAMP7 and Varp prevents SNARE complex formation by retaining VAMP7 in a fusogenically inactive state [40]. Most recently, Ripoll et al. have reported that a myosin VI motor binds to the neck of the VAMP7 tubule emanated from melanosomes and scissors it, although the molecular mechanism by which myosin VI is recruited to melanosomes still remains 
unknown [41]. Based on all of the above taken together, the following VAMP7 recovery model has been proposed (Figure 1): activated Rab32/Rab38 are recruited to melanosomes in a BLOC-3-dependent manner and simultaneously recruit VAMP7 in a BLOC-1-dependent manner, which enables formation of the Rab32/Rab38-Varp-VAMP7 complex on melanosomes. Then, the fusogenically inactive VAMP7 in this complex returns to early endosomes without interacting with its cognate $t$-SNAREs. Such a model, in which the BLOC-1 system and the BLOC-3 system function in opposite directions with respect to melanosome cargo transport, seems to be very attractive in the field of pigment biology. However, how this complex forms the VAMP7 recycling tubule from melanosomes is unknown, even though it has recently been reported that myosin VI regulates the formation of VAMP7-tubules. Clarifying the interaction between this complex and myosin VI and its regulatory mechanism is the next important issue that needs to be addressed in future studies.

\section{Concluding remarks}

In this article, we have summarized the proposed functions of SNAREs in melanocytes, especially in regard to the fusion processes between melanosome-cargo-containing endosomes and melanosomes. At least two types of SNARE complexes control the fusion processes that deliver melanosome cargoes into melanosomes, but we still cannot answer the basic question: How do melanocytes use two different SNARE mechanisms in melanosome cargo transport? Future extensive research will clarify the difference 
between the functions of syntaxin-3 and syntaxin-13 in melanosome cargo transport. Moreover, although the discovery of a SNARE recycling system from mature melanosomes is intriguing, several important questions regarding to the recycling system have arisen. How does new tubulation from mature melanosomes occur? Does VAMP7 actually return to early endosomes? Is the SNARE recycling system generally present in mammalian cells, despite the fact that expression of Rab32/Rab38 are cell-type specific [42]? Moreover, since the Rab32/Rab38 activation cycle is likely to be related to the SNARE recycling system, it would also be very interesting to clarify the relationship between BLOC-3 (Rab32/Rab38-GEF) and RUTBC1 (Rab32/Rab38-GAP [GTPase-activating protein]) [43]. Thus, the discovery of the SNARE recovery system on melanosomes may provide a new starting point for investigation of the precise molecular mechanism of melanosome cargo transport to melanosomes.

\section{Acknowledgements}

This work was supported in part by Grant-in-Aid for Scientific Research(C) from the Ministry of Education, Culture, Sports, Science and Technology (MEXT) of Japan (grant number 15K07039 to N.O.), Grant-in-Aid for Scientific Research on Innovative Areas from MEXT (grant numbers $17 \mathrm{H} 05682$ to M.F.), by Japan Science and Technology Agency (JST) CREST (grant Number JPMJCR17H4 to M.F.), and by Hoyu Science Foundation (to M.F.). 


\section{References}

1. Ohbayashi, N. and Fukuda, M. (2012) Role of Rab family GTPases and their effectors in melanosomal logistics. J. Biochem. 151, 343-351.

2. Wei, A.H. and Li, W. (2013) Hermansky-Pudlak syndrome: pigmentary andnon-pigmentary defects and their pathogenesis. Pigment. Cell Melanoma Res. 26, 176-192.

3. Fukuda, M. (2016) Lysosome-related organelles. Encyclopedia of Cell Biology (Bradshaw R. A. and Stahl P. D ed.) 2, 235-242.

4. Salazar, G., Craige, B., Styers, M.L., Newell-Litwa, K.A., Doucette, M.M., Wainer, B.H., Falcon-Perez, J.M., Dell'Angelica, E.C., Peden, A.A., Werner, E. et al. (2006) BLOC-1 complex deficiency alters the targeting of adaptor protein complex-3 cargoes. Mol. Biol. Cell 17, 4014-4026.

5. Di Pietro, S.M., Falcon-Perez, J.M., Tenza, D., Setty, S.R.G., Marks, M.S., Raposo, G. and Dell'Angelica, E.C. (2006) BLOC-1 interacts with BLOC-2 and the AP-3 complex to facilitate protein trafficking on endosomes. Mol. Biol. Cell 17, 4027-4038.

6. Gautam, R., Novak, E.K., Tan, J., Wakamatsu, K., Ito, S. and Swank, R.T. (2006) Interaction of Hermansky-Pudlak Syndrome genes in the regulation of lysosome-related organelles. Traffic 7, 779-792.

7. Setty, S.R.G., Tenza, D., Sviderskaya, E.V., Bennett, D.C., Raposo, G. and Marks, M.S. (2008) Cell-specific ATP7A transport sustains copper-dependent tyrosinase activity in melanosomes. Nature 454, 1142-1146.

8. Setty, S.R.G., Tenza, D., Truschel, S.T., Chou, E., Sviderskaya, E.V., Theos, A.C., Lamoreux, M.L., Di Pietro, S.M., Starcevic, M., Bennett, D.C. et al. (2007) BLOC-1 is required for cargo-specific sorting from vacuolar early endosomes toward lysosome-related organelles. Mol. Biol. Cell 18, 768-780.

9. Cheli, V.T., Daniels, R.W., Godoy, R., Hoyle, D.J., Kandachar, V., Starcevic, M., Martinez-Agosto, J.A., Poole, S., DiAntonio, A., Lloyd, V.K. et al. (2010) Genetic modifiers of abnormal organelle biogenesis in a Drosophila model of BLOC-1 deficiency. Hum. Mol. Genet. 19, 861-878. 
10. Delevoye, C., Heiligenstein, X., Ripoll, L., Gilles-Marsens, F., Dennis, M.K., Linares, R.A., Derman, L., Gokhale, A., Morel, E., Faundez, V. et al. (2016) BLOC-1 brings together the actin and microtubule cytoskeletons to generate recycling endosomes. Curr. Biol. 26, 1-13.

11. Dennis, M.K., Mantegazza, A.R., Snir, O.L., Tenza, D., Acosta-Ruiz, A., Delevoye, C., Zorger, R., Sitaram, A., de Jesus-Rojas, W., Ravichandran, K. et al. (2015) BLOC-2 targets recycling endosomal tubules to melanosomes for cargo delivery. J. Cell Biol. 209, 563-577.

12. Sitaram, A., Piccirillo, R., Palmisano, I., Harper, D.C., Dell'Angelica, E.C., Schiaffino, M.V. and Marks, M.S. (2009) Localization to mature melanosomes by virtue of cytoplasmic dileucine motifs is required for human OCA2 function. Mol. Biol. Cell 20, 1464-1477.

13. Huizing, M., Sarangarajan, R., Strovel, E., Zhao, Y., Gahl, W.A. and Boissy, R.E. (2001) AP-3 mediates tyrosinase but not TRP-1 trafficking in human melanocytes. Mol. Biol. Cell 12, 2075-2085.

14. Theos, A.C., Tenza, D., Martina, J.A., Hurbain, I., Peden, A.A., Sviderskaya, E.V., Stewart, A., Robinson, M.S., Bennett, D.C., Cutler, D.F. et al. (2005) Functions of adaptor protein (AP)-3 and AP-1 in tyrosinase sorting from endosomes to melanosomes. Mol. Biol. Cell 16, 5356-5372.

15. McNew, J.A, Parlati, F., Fukuda, R., Johnston, R.J., Paz, K., Paumet, F., Söllner, T.H. and Rothman, J.E. (2000) Compartmental specificity of cellular membrane fusion encoded in SNARE proteins. Nature 407, 153-159.

16. Sutton, R.B., Fasshauer, D., Jahn, R. and Brunger, A.T. (1998) Crystal structure of a SNARE complex involved in synaptic exocytosis at $2.4 \AA$ resolution. Nature 395, 347-353.

17. Scott, G. and Zhao, Q. (2001) Rab3a and SNARE proteins: potential regulators of melanosome movement. J. Invest. Dermatol. 116, 296-304.

18. Wade, N., Bryant, N.J., Connolly, L.M., Simpson, R.J., Luzio, J.P., Piper, R.C. and James, D.E. (2001) Syntaxin 7 complexes with mouse Vps10p tail interactor $1 \mathrm{~b}$, syntaxin 6, vesicle-associated membrane protein (VAMP)8, and VAMP7 in B16 melanoma cells. J. Biol. Chem. 276, 19820-19827. 
19. Dennis, M.K., Delevoye, C., Acosta-Ruiz, A., Hurbain, I., Romao, M., Hesketh, G.G., Goff, P.S., Sviderskaya, E.V., Bennett, D.C., Luzio, J.P. et al. (2016) BLOC-1 and BLOC-3 regulate VAMP7 cycling to and from melanosomes via distinct tubular transport carriers. J. Cell Biol. 214, 293-308.

20. Tamura, K., Ohbayashi, N., Ishibashi, K. and Fukuda, M. (2011) Structure-function analysis of VPS9-ankyrin-repeat protein (Varp) in the trafficking of tyrosinase-related protein 1 in melanocytes. J. Biol. Chem. 286, 7507-7521.

21. Jani, R.A., Purushothaman, L.K., Rani, S., Bergam, P. and Setty, S.R.G. (2015) STX13 regulates cargo delivery from recycling endosomes during melanosome biogenesis. J. Cell Sci. 128, 3263-3276.

22. Yatsu, A., Ohbayashi, N., Tamura, K. and Fukuda, M. (2013) Syntaxin-3 is required for melanosomal localization of Tyrp1 in melanocytes. J. Invest. Dermatol. 133, 2237-2246.

23. Rosengren Pielberg, G., Golovko, A., Sundstrom, E., Curik, I., Lennartsson, J., Seltenhammer, M.H., Druml, T., Binns, M., Fitzsimmons, C., Lindgren, G. et al. (2008) A cis-acting regulatory mutation causes premature hair graying and susceptibility to melanoma in the horse. Nat. Genet. 40, 1004-1009.

24. Kean, M.J., Williams, K.C., Skalski, M., Myers, D., Burtnik, A., Foster, D. and Coppolino, M.G. (2009) VAMP3, syntaxin-13 and SNAP23 are involved in secretion of matrix metalloproteinases, degradation of the extracellular matrix and cell invasion. J. Cell Sci. 122, 4089-4098.

25. McBride, H.M., Rybin, V., Murphy, C., Giner, A., Teasdale, R. and Zerial, M. (1999) Oligomeric complexes link Rab5 effectors with NSF and drive membrane fusion via interactions between EEA1 and syntaxin 13. Cell 98, 377-386.

26. Moriyama, K. and Bonifacino, J.S. (2002) Pallidin is a component of a multi-protein complex involved in the biogenesis of lysosome-related organelles. Traffic 3, 666-677.

27. Chung, W.Y., Park, H.W., Han, J.W., Lee, M.G. and Kim, J.Y. (2013) WNK4 inhibits plasma membrane targeting of NCC through regulation of syntaxin13 
SNARE formation. Cell Signal. 25, 2469-2477.

28. Chi, A., Valencia, J.C., Hu, Z.Z., Watabe, H., Yamaguchi, H., Mangini, N.J., Huang, H., Canfield, V.A., Cheng, K.C., Yang, F. et al. (2006) Proteomic and bioinformatic characterization of the biogenesis and function of melanosomes. J. Proteome Res. 5, 3135-3144.

29. Fukuda, M. (2008) Regulation of secretory vesicle traffic by Rab small GTPases. Cell. Mol. Life Sci. 65, 2801-2813.

30. Loftus, S.K., Larson, D.M., Baxter, L.L., Antonellis, A., Chen, Y., Wu, X., Jiang, Y., Bittner, M., Hammer, J.A., III. and Pavan, W.J. (2002) Mutation of melanosome protein RAB38 in chocolate mice. Proc. Natl. Acad. Sci. U.S.A. 99, 4471-4476.

31. Bultema, J.J., Boyle, J.A., Malenke, P.B., Martin, F.E., Dell'Angelica, E.C., Cheney, R.E. and Di Pietro, S.M. (2014) Myosin Vc interacts with Rab32 and Rab38 proteins and works in the biogenesis and secretion of melanosomes. J. Biol. Chem. 289, 33513-33528.

32. Tamura, K., Ohbayashi, N., Maruta, Y., Kanno, E., Itoh, T. and Fukuda, M. (2009) Varp is a novel Rab32/38-binding protein that regulates Tyrp1 trafficking in melanocytes. Mol. Biol. Cell 20, 2900-2908.

33. Wasmeier, C., Romao, M., Plowright, L., Bennett, D.C., Raposo, G. and Seabra, M.C. (2006) Rab38 and Rab32 control post-Golgi trafficking of melanogenic enzymes. J. Cell Biol. 175, 271-281.

34. Fukuda, M. (2016) Multiple roles of VARP in endosomal trafficking: Rabs, retromer components and R-SNARE VAMP7 meet on VARP. Traffic 17, 709-719.

35. Marubashi, S., Ohbayashi, N. and Fukuda, M. (2016) A Varp-binding protein, RACK1, regulates dendrite outgrowth through stabilization of Varp protein in mouse melanocytes. J. Invest. Dermatol. 136, 1672-1680.

36. Yatsu, A., Shimada, H., Ohbayashi, N. and Fukuda, M. (2015) Rab40C is a novel Varp-binding protein that promotes proteasomal degradation of Varp in melanocytes. Biol. Open 4, 267-275. 
37. Burgo, A., Sotirakis, E., Simmler, M.C., Verraes, A., Chamot, C., Simpson, J.C., Lanzetti, L., Proux-Gillardeaux, V. and Galli, T. (2009) Role of Varp, a Rab21 exchange factor and TI-VAMP/VAMP7 partner, in neurite growth. EMBO Rep. 10, 1117-1124.

38. Pryor, P.R., Jackson, L., Gray, S.R., Edeling, M.A., Thompson, A., Sanderson, C.M., Evans, P.R., Owen, D.J. and Luzio, J.P. (2008) Molecular basis for the sorting of the SNARE VAMP7 into endocytic clathrin-coated vesicles by the ArfGAP Hrb. Cell 134, 817-827.

39. Gerondopoulos, A., Langemeyer, L., Liang, J.R., Linford, A. and Barr, F.A. (2012) BLOC-3 mutated in Hermansky-Pudlak syndrome is a Rab32/38 guanine nucleotide exchange factor. Curr. Biol. 22, 2135-2139.

40. Schäfer, I.B., Hesketh, G.G., Bright, N.A., Gray, S.R., Pryor, P.R., Evans, P.R., Luzio, J.P. and Owen, D.J. (2012) The binding of Varp to VAMP7 traps VAMP7 in a closed, fusogenically inactive conformation. Nat. Struct. Mol. Biol. 19, 1300-1309.

41. Ripoll, L., Heiligenstein, X., Hurbain, I., Domingues, L., Figon, F., Petersen, K.J., Dennis, M.K., Houdusse, A., Marks, M.S., Raposo, G. et al. (2018) Myosin VI and branched actin filaments mediate membrane constriction and fission of melanosomal tubule carriers. J. Cell Biol. doi: 1083/jcb.201709055.

42. Ohbayashi, N., Fukuda, M., and Kanaho, Y. (2017) Rab32 subfamily small GTPases: pleiotropic Rabs in endosomal trafficking. J. Biochem. 162, 65-71.

43. Marubashi, S., Shimada, H., Fukuda, M., and Ohbayashi, N. (2016) RUTBC1 functions as a GTPase-activating protein for Rab32/38 and regulates melanogenic enzyme trafficking in melanocytes. J. Biol. Chem. 291, 1427-1440. 


\section{Figure legends}

Figure 1 Proposed roles of SNAREs and their regulators in melanosome biogenesis

Melanosome biogenesis progresses as melanosome cargoes such as tyrosinase and Tyrp1 are transported to melanosomes. Tyrp1 and tyrosinase are transported from early endosomes to melanosomes (more specifically, to immature melanosomes) by a BLOC-1-dependent pathway and an AP-3-dependent pathway, respectively. The BLOC-1 pathway loads cargoes, including Tyrp1, into recycling endosomes, and then BLOC-2 promotes their fusion with melanosomes. In the BLOC-1/BLOC-2-dependent pathway, syntaxin-13, which is expressed in pan-endosomes, is transported together with Tyrp1 in the recycling endosomes, and then forms a complex with VAMP7 localized at melanosomes that delivers Tyrp1 to the melanosomes. Meanwhile, syntaxin-3 and SNAP-23 are localized at melanosomes and regulate transport of melanosome cargoes by forming a complex with VAMP7 transported by endosomes (anterograde route). Both VAMP7-syntaxin-13-unknown-t-SNARE complex and VAMP7-syntaxin-3-SNAP23 complex are likely to be involved in the fusion of Tyrp1-containing vesicle/tubule with immature melanosomes. Rab32/Rab38-Varp-VAMP7 complex also participates in the anterograde route of the melanosome cargoes upstream of the SNARE comlexes. VAMP7 needs to be released from the negative regulation by Rab32/Rab38-Varp in the vicinity of melanosomes to exert its fusogenic activity (i.e., formation of the VAMP7-syntaxin-3-SNAP23 complex and VAMP7-syntaxin-13-unknown-t-SNARE 
complex.) After melanosome maturation, Rab32/Rab38 are activated by BLOC-3 and recruited to mature melanosomes. Then, Rab32/Rab38 promote recruitment of Varp there and form a complex consisting of Rab32/Rab38-Varp-VAMP7 that suppresses the fusogenic activity of VAMP7. Finally, VAMP7 tubules are formed at the pole of melanosomes in myosin VI-dependent manner (retrograde route) and the tubules presumably mediate transport of VAMP7 to early endosomes. The dotted arrows indicate as yet unidentified transport pathways. 


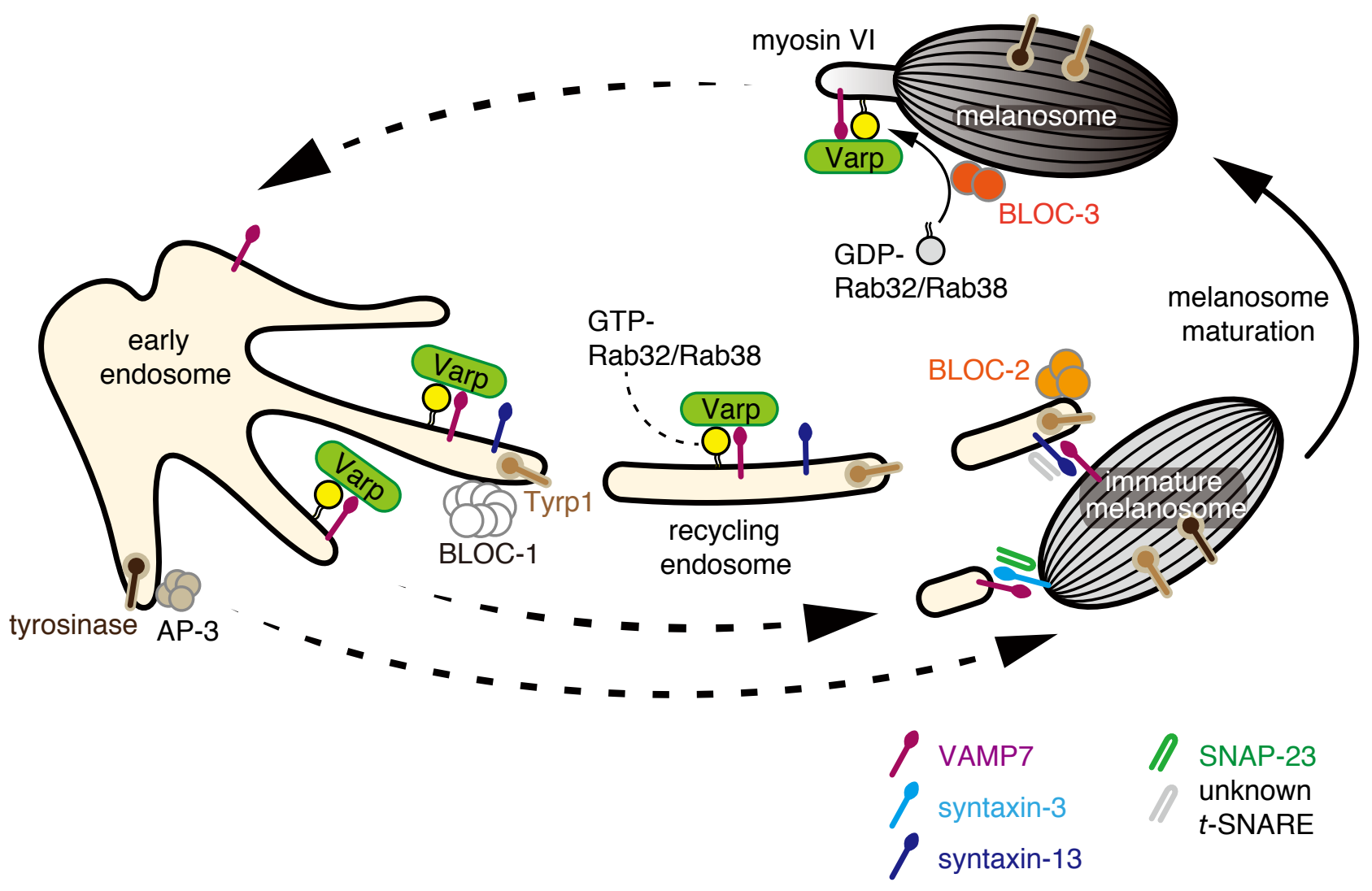

Figure 1 